University of Massachusetts Amherst

ScholarWorks@UMass Amherst

Astronomy Department Faculty Publication Series

Astronomy

2005

\title{
Highly clumpy structure of the thermal composite supernova remnant 3C 391 unveiled by Chandra
}

Y Chen

$\mathrm{YSu}$

PO Slane

QD Wang

University of Massachusetts - Amherst

Follow this and additional works at: https://scholarworks.umass.edu/astro_faculty_pubs

Part of the Astrophysics and Astronomy Commons

\section{Recommended Citation}

Chen, Y; Su, Y; Slane, PO; and Wang, QD, "Highly clumpy structure of the thermal composite supernova remnant 3C 391 unveiled by Chandra" (2005). Advances in Space Research. 1019.

10.1016/j.asr.2005.01.005

This Article is brought to you for free and open access by the Astronomy at ScholarWorks@UMass Amherst. It has been accepted for inclusion in Astronomy Department Faculty Publication Series by an authorized administrator of ScholarWorks@UMass Amherst. For more information, please contact scholarworks@library.umass.edu. 


\title{
Highly Clumpy Structure of the Thermal Composite Supernova Remnant 3C 391 Unveiled by Chandra*
}

\author{
Yang Chen ${ }^{1}$, Yang $\mathrm{Su}^{1}$, Patrick O. Slane ${ }^{3}$, Q. Daniel Wang ${ }^{2}$
}

\begin{abstract}
The nature of the internal thermal X-ray emission seen in "thermal composite" supernova remnants is still uncertain. Chandra observation of the 3C391 shows a southeast-northwest elongated morphology and unveils a highly clumpy structure of the remnant. Detailed spatially resolved spectral analysis for the small-scale features reveals normal metal abundance and uniform temperature for the interior gas. The properties of the hot gas comparatively favor the cloudlet evaporation model as a main mechanism for the "thermal composite" X-ray appearance, though radiative rim and thermal conduction may also be effective. A faint protrusion is found in $\mathrm{Si}$ and $\mathrm{S}$ lines out of the southwest radio border.
\end{abstract}

Key words: radiation mechanisms: thermal, supernova remnants: individual:

3C 391 (G31.9+0.0), X-rays: ISM

PACS: $95.30 . \mathrm{Gv}, 98.38 . \mathrm{Mz}, 95.85 . \mathrm{Nv}$

\section{Introduction}

3C 391 (G31.9+0.0), with irregular morphology (e.g. Chen \& Slane 2001), has been classified into the "thermal composite" category of supernova remnants (SNRs) (Rho \& Petre 1998). They generate bright thermal X-ray emission interior to their radio shells, and have faint X-ray rims. They are usually found to interact with adjacent molecular clouds, characterized by the hydroxyl radical maser emission (e.g. Yusef-Zadeh et al. 2003). The nature of the internal thermal X-ray emission seen in mixed morphology remnants is still uncertain.

\footnotetext{
* Supported by NSFC grants 10073003 \& 10221001, CMST grant nkbrsf-g19990754, NASA contract NAS8-39073, and NASA grants GO2-3081X \& NAG5-8935.

1 Department of Astronomy, Nanjing University, Nanjing 210093, P.R.China

2 Harvard-Smithsonian Center for Astrophys., Cambridge, MA 02138

3 Dept. of Astr., B619E-LGRT, Univ. of Massachusetts, Amherst, MA01003
} 
So far at least four candidate scenarios compete to account for centrallybrightened X-ray morphology. The first scenario is radiative cooling of the rim gas. Under this hypothesis, the gas at the rim has been cooled down in the radiative stage, with a temperature so low that its X-ray emission is very weak, while the gas in the inner volume is still hot enough to emit strong X-rays (e.g. Rho \& Petre 1998). The second mechanism invokes thermal conduction. It is suggested that thermal conduction in the remnant can prevent formation of the very tenuous, hot gas in the inner part and therefore change the interior structure from the standard Sedov solution, resulting in a nonnegligible density and luminous X-ray in the interior (as observed in, especially, the radiative stage) (Cox et al. 1999). The third scenario is cloudlet evaporation in the SNR interior. When an SNR expands in an inhomogeneous interstellar medium (ISM) whose mass is mostly contained in small clouds, the clouds engulfed by the blast wave can be evaporated to gradually increase the density of the interior gas; consequently, the SNR appears internally X-ray brightened (White \& Long 1991). The fourth suggestion is that the mixed morphology is a projection effect. For shell-like SNRs that evolve in a density gradient such as at the edge of a molecular cloud, if the line of sight is essentially aligned with the density gradient as well as the magnetic field, the SNRs will appear as thermal composites (Petruk 2001). Here we report on high resolution Chandra observation of 3C 391 and compare the properties with these scenarios.

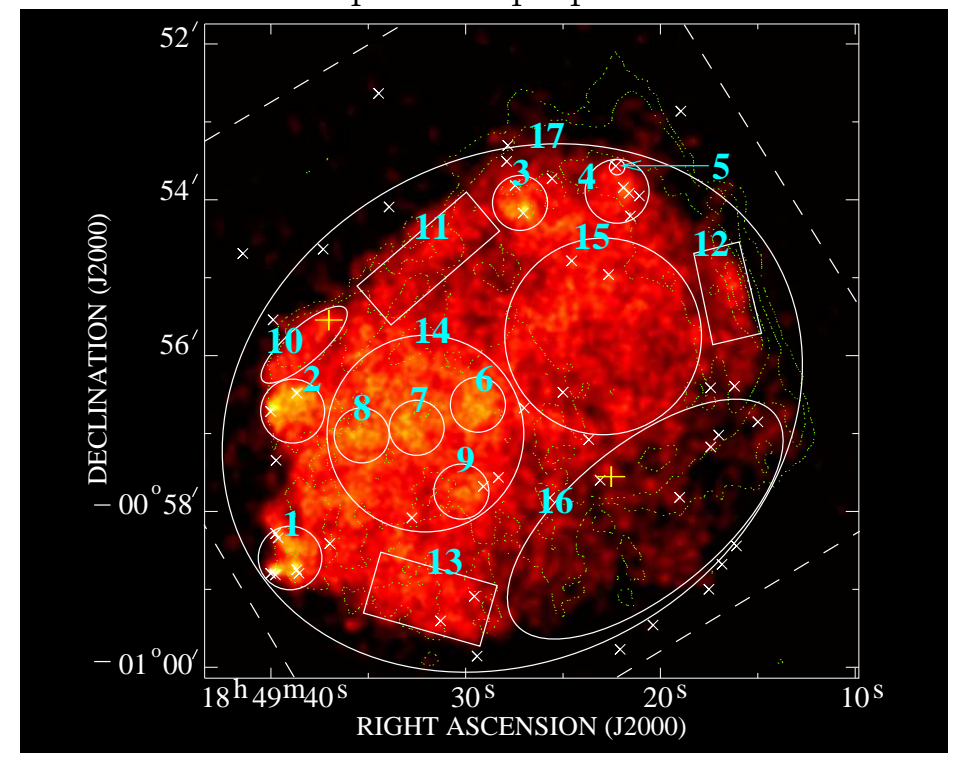

Fig. 1 Smoothed diffuse emission from SNR 3C 391 in the broad band 0.3-7.0 keV (with count-to-noise ratio of 6). The color is logarithmically scaled in the range $(1.19-331.85) \times 10^{-2} \mathrm{ct} \mathrm{s}^{-1} \mathrm{arcmin}^{-2}$. The location of the sources removed from the data before the smoothing are marked by white cross labels. All the regions used for spectral analysis are indicated in white, with cyan numerical labels. The overlaid $1.5 \mathrm{GHz}$ radio contours (in green) are at 1.5, 4.5, 13.6, 28.8, and $50 \times 10^{-3} \mathrm{Jy} \mathrm{beam}^{-1}$ (Moffett \& Reynolds 1994). The two yellow plus signs denote the OH maser points (Frail et al. 1996). The white dashed lines denote the border of the S3 chip. 


\section{Data and analysis}

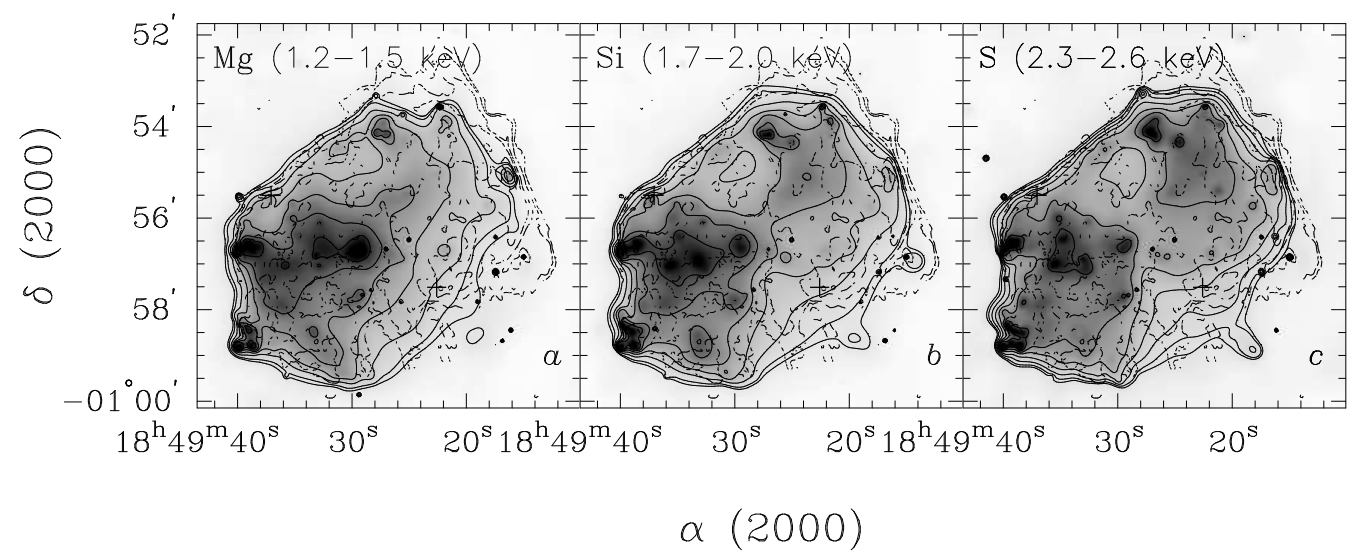

Fig. 2 Smoothed narrow band 1.2-1.5, 1.7-2.0, and 2.3-2.6 keV (including $\mathrm{Mg} \mathrm{He} \alpha$, Si He $\alpha$, and S He $\alpha$, respectively) diffuse emission images (with $\mathrm{S} / \mathrm{N}$ ratio of 3) overlaid with the dashed contours of $1.5 \mathrm{GHz}$ radio emission (at 1.5, 3.2, 8.25, 16.7, 43.7, and $62.2 \times 10^{-3} \mathrm{Jy}_{\text {beam }}{ }^{-1}$ ) (Moffett \& Reynolds 1994). The seven levels of solid contours are plotted with square-root intensity scales between the maximum and the $15 \%$ maximum brightness. The two plus signs in each panel denote the $\mathrm{OH}$ maser points.

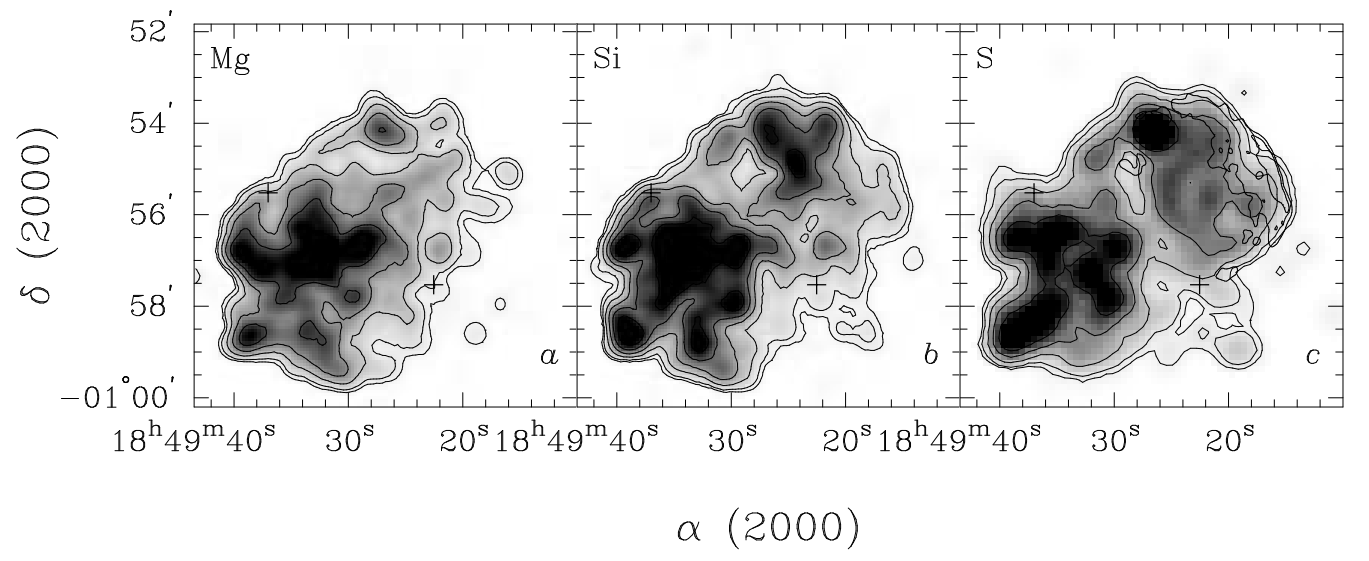

Fig. $3 \mathrm{EW}$ images of $\mathrm{Mg}, \mathrm{Si}$, and $\mathrm{S}$ lines. The $\mathrm{Mg}$ and $\mathrm{Si}(\mathrm{S})$ images are extracted with 4" (8") pixels and smoothed by a Gaussian with $\sigma=12$ " (16"). The seven levels of solid contours are plotted with square-root intensity scales between the maximum and the $3 \%$ maximum brightness. The two plus signs in each panel denote the $\mathrm{OH}$ maser points.

SNR 3C 391 was observed with the Chandra ACIS on 03 August 2002. The level 1 raw event data were reprocessed to generate a level 2 event file using the CIAO software package (version 2.3), resulting a net $60.7 \mathrm{ks}$ exposure. In Figures 1 and 2, we show the diffuse X-ray map in $0.3-7 \mathrm{keV}$ and the narrow band 1.2-1.5, 1.7-2.0, and 2.3-2.6 keV (including $\mathrm{Mg} \mathrm{He} \alpha$, Si He $\alpha$, and $\mathrm{S} \mathrm{He} \alpha$, respectively) diffuse emission images. The equivalent width maps (i.e., the line-to-continuum ratio maps) for $\mathrm{Mg}, \mathrm{Si}$, and $\mathrm{S}$ are shown in Figure 3. 
These X-ray images display the SE-NW elongated morphology, revealing a highly clumpy structure of the remnant, with clumps or knots located in both the SE and NW parts. Several remarkable, very bright knotty features appear on the east and SE border (regions \#1 and \#2) of 3C 391. These bright knots on the border may be small clouds that have recently been shocked by the supernova blast wave. A bright enhancement is peaked near the NW border (region \#3). A complex mixture of knots is seen in the SE part of the remnant interior, including at least four bright enhancements indicated as regions \#6, \#7, \#8, and \#9.

In Fig.1, arc- or shell-like structures are seen along the northeastern and northern rim (regions \#10 and \#11). An X-ray brightened slab at the west rim appears to be very close to (just slightly behind) the radio peak emission (region \#12), and may be related to a small dense region there. On the southwest, faint diffuse emission seems to extend out of the radio border. On the corresponding location in the narrow-band and EW images of Si and S lines (Figs.2b, 2c, 3b, and 3c), there is a finger-like feature protruding radially out of the radio border. This protrusion looks somewhat similar to the apparent protrusion in $\mathrm{Si}$ and $\mathrm{S}$ lines that was recently revealed on the northeastern border of Cas A and explained as one of the jets of ejecta (Hwang, Holt, \& Petre 2000; Hwang et al. 2004). However, the small number of counts collected for this feature makes it difficult to determine the metal abundances.

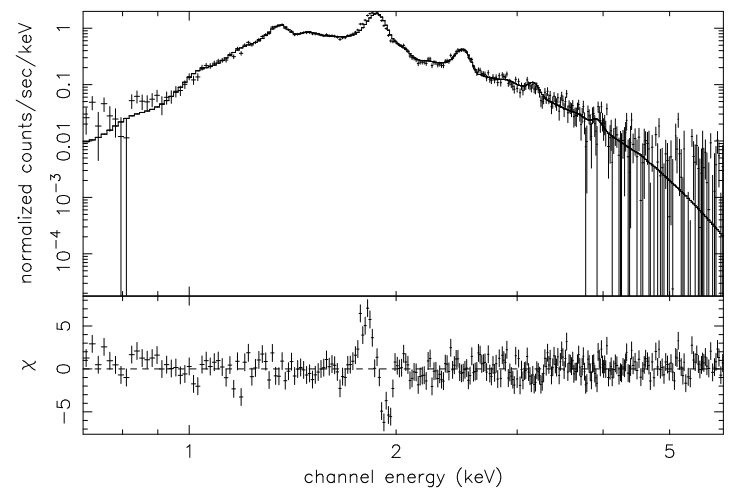

Fig. 4 Chandra ACIS spectra of the entire remnant of 3C 391

With the point-like sources removed, X-ray spectra were extracted from 17 regions shown in Fig.1. Most of the small-scale regions are chosen to include the small features of X-ray enhancement such as the knots and the faint shell like structures. The area on the S3 chip outside region \#17 was used for background.

The distinct line features, $\mathrm{Mg} \operatorname{He} \alpha(\sim 1.35 \mathrm{keV})$, Si He $\alpha(\sim 1.85 \mathrm{keV})$, and $\mathrm{S} \mathrm{He} \alpha(\sim 2.46 \mathrm{keV})$ in the spectra (see Fig.4) indicate the thermal origin of the diffuse gas. We find that the spectra of the diffuse gas can be best described by the VNEI model with the correction of interstellar absorption. The spectral fit results are tabulated in the Table.

The absorption column density is found to generally increase across the remnant from SE to NW, consistent with the existence of a molecular cloud to the NW (Wilner et al. 1998). The spectral fits show that the diffuse emission from various regions have ionization parameters $\left(n_{e} t\right)$ close to or higher than $10^{12} \mathrm{~cm}^{-3} \mathrm{~s}$. This implies that the hot plasma in the SNR is very close to, or 
is basically in, the ionization equilibrium. The spectral fits also show that the diffuse emission from various regions can be well fitted with solar abundances or abundances very close to solar values. The temperature of the gas interior to the SNR is generally $\sim 0.5-0.6 \mathrm{keV}$, with only small fluctuations. The gas density of each defined region was roughly estimated (as also listed in the Table). Apart from the compact knots on the SE and eastern boundary, most of the bright knots have a gas density $\sim 5-7 f^{-1 / 2} d_{8}^{-1 / 2} \mathrm{~cm}^{-3}$ and most of the regions along the remnant border have a density $\sim 1-3 f^{-1 / 2} d_{8}^{-1 / 2} \mathrm{~cm}^{-3}$ (where $d_{8}=d / 8 \mathrm{kpc}$ denotes the distance). The X-ray luminosity in $0.5-10 \mathrm{keV}$ of the remnant is $\sim 3.5 \times 10^{36} d_{8}^{2} \operatorname{ergs~s}^{-1}$.

VNEI fitting results with the $90 \%$ confidence ranges and density estimates

\begin{tabular}{|c|c|c|c|c|c|c|}
\hline regions & $\chi^{2} /$ d.o.f. & $\begin{array}{c}N_{\mathrm{H}} \\
\left(10^{22} \mathrm{~cm}^{-2}\right)\end{array}$ & $\begin{array}{c}k T_{x} \\
(\mathrm{keV})\end{array}$ & $\begin{array}{c}n_{e} t \\
\left(10^{11} \mathrm{~cm}^{-3} \mathrm{~s}\right)\end{array}$ & $\begin{array}{c}f n_{e} n_{\mathrm{H}} V / d_{8}^{2} \mathrm{a} \\
\left(10^{57} \mathrm{~cm}^{-3}\right)\end{array}$ & $\begin{array}{c}n_{\mathrm{H}} / f^{-1 / 2} d_{8}^{-1 / 2} \\
\left(\mathrm{~cm}^{-3}\right)\end{array}$ \\
\hline 1 & $90.5 / 65$ & $2.8 \pm 0.1$ & $0.67_{-0.04}^{+0.02}$ & $>200$ & $2.55_{-0.53}^{+0.27}$ & 18 \\
\hline 2 & $87.0 / 79$ & $2.7 \pm 0.1$ & $0.58_{-0.05}^{+0.06}$ & $5.1_{-1.3}^{+4.6}$ & $3.84_{-0.89}^{+1.33}$ & 11 \\
\hline 3 & $68.8 / 48$ & $3.4 \pm 0.2$ & $0.56_{-0.09}^{+0.06}$ & $>6.4$ & $3.46_{-0.90}^{+2.08}$ & 6.4 \\
\hline \multirow[t]{2}{*}{$4^{\mathrm{b}}$} & $108.9 / 79$ & $4.1_{-0.2}^{+0.3}$ & $0.62_{-0.05}^{+0.04}$ & $>30$ & $9.42_{-2.33}^{+3.10}$ & 4.8 \\
\hline & \multicolumn{6}{|c|}{$\left([\mathbf{M g} / \mathbf{H}]=1.23_{-0.44}^{+0.69},[\mathbf{S i} / \mathbf{H}]=0.66_{-0.15}^{+0.19},[\mathbf{S} / \mathbf{H}]=0.51_{-0.20}^{+0.22}\right)$} \\
\hline 6 & $65.2 / 58$ & $2.7_{-0.1}^{+0.2}$ & $0.56_{-0.06}^{+0.08}$ & $5.0_{-2.1}^{+3.8}$ & $3.20_{-0.98}^{+1.41}$ & 6.1 \\
\hline 7 & $57.8 / 58$ & $3.0_{-0.2}^{+0.1}$ & $0.54_{-0.06}^{+0.05}$ & $>5.5$ & $3.97_{-0.98}^{+1.65}$ & 6.8 \\
\hline 8 & $72.4 / 52$ & $2.9_{-0.1}^{+0.2}$ & $0.63_{-0.07}^{+0.08}$ & $>3.4$ & $2.52_{-0.65}^{+0.60}$ & 5.4 \\
\hline 9 & $58.2 / 43$ & $2.9 \pm 0.2$ & $0.63_{-0.11}^{+0.07}$ & $>2.7$ & $1.72_{-0.41}^{+1.35}$ & 4.5 \\
\hline 10 & $44.2 / 44$ & $3.0_{-0.1}^{+0.2}$ & $0.79_{-0.10}^{+0.14}$ & $3.1_{-1.4}^{+3.6}$ & $1.16_{-0.34}^{+0.52}$ & 2.3 \\
\hline 11 & $96.9 / 72$ & $2.8 \pm 0.1$ & $0.59_{-0.04}^{+0.06}$ & $>3.8$ & $3.17_{-0.77}^{+0.38}$ & 1.9 \\
\hline 12 & $49.8 / 32$ & $3.7_{-0.4}^{+0.6}$ & $0.58_{-0.12}^{+0.11}$ & $>265$ & $2.28_{-0.96}^{+3.19}$ & 2.0 \\
\hline 13 & $107.0 / 82$ & $3.0_{-0.1}^{+0.2}$ & $0.46_{-0.03}^{+0.04}$ & $>106$ & $8.23_{-2.12}^{+2.73}$ & 2.6 \\
\hline \multirow[t]{2}{*}{$14^{\mathrm{b}}$} & $273.6 / 180$ & $2.9 \pm 0.1$ & $0.55 \pm 0.02$ & $>9.0$ & $35.00_{-5.38}^{+3.75}$ & 2.9 \\
\hline & \multicolumn{6}{|c|}{$\left([\mathrm{Mg} / \mathrm{H}]=1.12_{-0.09}^{+0.13},[\mathrm{Si} / \mathrm{H}]=0.87_{-0.05}^{+0.08},[\mathrm{~S} / \mathrm{H}]=0.80_{-0.09}^{+0.13}\right)$} \\
\hline \multirow[t]{2}{*}{$15^{\mathrm{b}}$} & $237.8 / 163$ & $3.5 \pm 0.1$ & $0.54_{-0.01}^{+0.02}$ & $>23$ & $34.08_{-4.20}^{+5.42}$ & 2.8 \\
\hline & \multicolumn{6}{|c|}{$\left([\mathrm{Mg} / \mathrm{H}]=0.92_{-0.13}^{+0.14},[\mathrm{Si} / \mathrm{H}]=0.53 \pm 0.05,[\mathrm{~S} / \mathrm{H}]=0.64_{-0.11}^{+0.12}\right)$} \\
\hline \multirow[t]{2}{*}{$16^{\mathrm{b}}$} & $156.3 / 127$ & $3.2 \pm 0.2$ & $0.53_{-0.06}^{+0.04}$ & $>239$ & $10.0_{-2.60}^{+5.42}$ & 1.1 \\
\hline & \multicolumn{6}{|c|}{$\left([\mathrm{Mg} / \mathrm{H}]=0.93_{-0.26}^{+0.28},[\mathrm{Si} / \mathrm{H}]=0.54_{-0.11}^{+0.12},[\mathrm{~S} / \mathrm{H}]=0.54_{-0.21}^{+0.24}\right)$} \\
\hline \multirow[t]{2}{*}{$17^{\mathrm{b}}$} & $710.5 / 296$ & $3.1 \pm 0.1$ & $0.56 \pm 0.01$ & $>12.8$ & $150 \pm 10$ & 1.9 \\
\hline & \multicolumn{6}{|c|}{$\left([\mathrm{Mg} / \mathrm{H}]=0.97_{-0.05}^{+0.07},[\mathrm{Si} / \mathrm{H}]=0.70 \pm 0.03,[\mathrm{~S} / \mathrm{H}]=0.71_{-0.05}^{+0.06}\right)$} \\
\hline
\end{tabular}

a: $f$ is the filling factor of the hot gas.

b: Making abundances of $\mathrm{Mg}, \mathrm{Si}$, and $\mathbf{S}$ free parameters apparently improves the fit.

\section{Discussion on the composite appearance}

Here we briefly compare the four mechanisms mentioned in $\S 1$ with the properties found from our spatially-resolved spectral analysis. 
i) Projection Effect The molecular cloud is located in the NW but the Xray emission is enhanced not only in the NW half, but also in the SE half. Additionally, the variation of the hydrogen column density across the remnant implies that the density gradient of the ambient medium seems to be close to the projection plane. Therefore, the projection effect does not match the observational properties. ii) Radiative Rim The filamentary near-infrared [Fe II] and the mid-infrared 12-18 $\mu \mathrm{m}$ [Ne II] and [Ne III] emission along the NW radio shell provide some evidence for a radiative cooling at the rim (Reach, Rho, \& Jarrett 2002). Along the NW border, the shell formation time is $t_{\text {shell }} \approx$ $3.8 \times 10^{3} E_{51}^{3 / 14} \mathrm{yr}$, comparable to remnant's age $t=(2 / 5)\left(r_{s} / v_{s}\right) \sim 4 \times 10^{3} \mathrm{yr}$. However the X-ray emission along the border (e.g. regions \#10, \#11, \#12, and \#16), which arises from hot gas $\left(\sim 7 \times 10^{6} \mathrm{~K}\right)$, indicates that a considerable amount of gas at the blast shock has not yet suffered significant radiative cooling. Additionally, the rim cooling mechanism can not explain what the central bright clumpy emission is. iii) Thermal Conduction For 3C 391, the thermal conduction scenario is favored by the radiative filaments along the NW border and the high ionization timescale implicative of relatively little newly shocked material. The conduction timescale $t_{\text {cond }} \sim 5.2 \times 10^{3}(\ell / 2.3 \mathrm{pc})^{2} \mathrm{yr}$ for the spatial scale $\ell$ of order the separation between clumps (typified by $\left.1^{\prime} \sim 2.3 \mathrm{pc}\right)$ is comparable to the remnant's age and hence implies a role of conduction in smoothing the interior temperature profile. However, the thermal conduction scenario asks for a decrease of temperature and an increase of gas density with remnant radius. This predicted behavior is inconsistent with the uniform distribution of the gas temperature and density. iv) Cloudlet Evaporation The relatively uniform distribution of temperature (even with slightly lower values at the center) is actually expected by the White \& Long (1991) cloudlet evaporation model for model parameters $\tau \rightarrow \infty$ and $C / \tau \gtrsim 3$, where $\tau$ is the ratio of the cloud evaporation timescale to the SNR's age and $C$ is the ratio of the mass in the cloudlets to the mass of ICM. The ratio between the mean density $\left(\sim 2 f^{-1 / 2} d_{8}^{-1 / 2} \mathrm{~cm}^{-3}\right)$ and the density along the border $\left(\sim 1-3 f^{-1 / 2} d_{8}^{-1 / 2} \mathrm{~cm}^{-3}\right)$ is basically consistent with that predicted in the evaporation model. In fact, the highly clumpy structure unveiled in this observation lends support to the conjecture that the ambient molecular cloud is inhomogeneous. A combination of very dense clumps and moderately dense gas are directly observed in the millimeter molecular lines (Reach \& Rho 1999). The cloudlets engulfed by the supernova blast wave can act as a large reservoir of interior gas by gradual evaporation. This could also explain why the interior gas density is much lower than that of the ambient cloud gas $\left(\sim 30-100 \mathrm{~cm}^{-3}\right)$. With this model, the supernova explosion energy is $E \sim 0.3-$ $1.4 \times 10^{51} d_{8}^{3}\left(n_{0} / 0.3 \mathrm{~cm}^{-3}\right)$ ergs. The main difficulty with the model is the high ionization age $\left(>10^{12} \mathrm{~s}\right)$ in some regions (e.g. region \#1) opposed to the low age of the newly evaporated gas. 


\section{References}

Chen, Y. \& Slane, P.O. ASCA Observations of the Thermal Composite Supernova Remnant 3C 391, 2001, ApJ, 563, 202-208

Cox, D. P., Shelton, R. L., Maciejewski, W., Smith, R. K., Plewa, T., Pawl, A., \& Rózyczka, M., Modeling W44 as a supernova remnant in a density gradient with a partially formed dense shell and thermal conduction in the hot interior. I. The analytical model, 1999, ApJ, 524, 179-191

Frail, D.A., Goss, W.M., Reynoso, E.M., Giacani, E.B., Green, A.J., \& Otrupcek, R., A survey for $\mathrm{OH}(1720 \mathrm{MHz})$ maser emission toward supernova remnants, 1996, AJ, 111, 1651-1659

Hwang, U., Holt, S.S., \& Petre, R., Mapping the X-ray-emitting ejecta in Cassiopeia A with Chandra, 2000, ApJ, 537, L119-L122

Hwang, U. et al., A million second Chandra view of Cassiopeia A, 2004, ApJ, 615, L117-L120

Moffett, D. A., \& Reynolds, S. P., Multifrequency studies of bright radio supernova remnants. 1: 3C 391, 1994, ApJ, 425, 668-686

Petruk, O., Thermal X-ray composites as an effect of projection, 2001, A\&A, $371,267-273$

Reach, W.T. \& Rho, J.H., Excitation and disruption of a giant molecular cloud by the supernova remnant 3C 391, 1999, ApJ, 511, 836-846

Reach, W.T., Rho, J.H., \& Jarrett, T.H., Molecular and Ionic Shocks in the Supernova Remnant 3C 391, 2002, ApJ, 564, 302-316

Rho, J.H., \& Petre, R., Mixed-morphology supernova remnants, 1998, ApJ, 503, L167-L170

White, R.L., \& Long, K.S., Supernova remnant evolution in an interstellar medium with evaporating clouds, 1991, ApJ, 373, 543-555

Wilner, D.J., Reynolds, S.P., \& Moffett, D.A., CO observations toward the supernova remnant 3C 391, 1998, ApJ, 115, 247-251

Yusef-Zadeh, F., Wardle, M., Rho, J., \& Sakano, M. OH (1720 MHz) Masers and mixed-morphology supernova remnants, ApJ, 2003, 585, 319-323 